\title{
1,2-Difunctionalized Bicyclo[1.1.1]pentanes: Long Sought After Bioisosteres for ortho/meta-Substituted Arenes
}

\author{
Jin-Xin Zhao, ${ }^{1,2}$ Yuxuan Chang, ${ }^{1}$ Jeff Elleraas, ${ }^{3}$ T. Patrick Montgomery, ${ }^{3}$ Jillian E. Spangler, ${ }^{3}$ Sajiv \\ K. Nair, ${ }^{3}$ Matthew Del Bel, ${ }^{3}$ Gary M. Gallego, ${ }^{3}$ James J. Mousseau, ${ }^{4}$ Matthew A. Perry, ${ }^{3}$ Michael \\ R. Collins, ${ }^{3}$ Julien C. Vantourout, ${ }^{1}$ and Phil S. Baran. ${ }^{*}$
}

1Department of Chemistry, The Scripps Research Institute, 10550 North Torrey Pines Road, La Jolla, California 92037, United States.

2State Key Laboratory of Drug Research, Shanghai Institute of Materia Medica, Chinese Academy of Sciences, 555 Zuchongzhi Road, Shanghai 201203, People's Republic of China.

3Department of Chemistry, La Jolla Laboratories, Pfizer, 10770 Science Center Drive, San Diego, California 92121, United States.

4Discovery Sciences, Medicine Design, Pfizer Global Research and Development, 445 Eastern Point Road, Groton, Connecticut, 06340, United States.

\begin{abstract}
The development of a versatile platform for the synthesis of 1,2-difunctionalized bicyclo[1.1.1]pentanes to potentially mimic ortho/meta-substituted arenes is described. The synthesis of useful building blocks bearing alcohol, amine, and carboxylic acid functional handles has been achieved from a simple common intermediate. Several ortho and/or meta-substituted benzene analogues as well as simple molecular matched pairs have also been prepared using this platform. In-depth biological and computational studies are currently in progress to validate the ortho and/or meta-character of these new bioisosteres. Results of these investigations will be reported in due course.
\end{abstract}

Main text: Over the past decade, medicinal chemist's mission to "escape from flatland" has altered the way synthesis in drug discovery is conducted. ${ }^{1}$ Specifically, $\mathrm{C}\left(\mathrm{sp}^{3}\right)$-rich structures, where the venerable benzene ring is replaced with saturated bioisosteres, have started to rapidly populate the patent literature, compound libraries, and the toolbox of medicinal chemistry tactics. ${ }^{1}$ These 3D-moieties often exhibit improved biological activities, physicochemical properties and metabolic profiles compared to the parent benzene ring. ${ }^{2}$ These singular characteristics have encouraged several academic and industrial groups, including the Pfizer/Scripps team, to achieve the functionalization of these small structures. ${ }^{3}$ As a subset of these bioisosteres, 
bicyclo[1.1.1]pentane (BCP) cores have been extensively studied and proven valuable alternatives to benzene rings. ${ }^{3}$ However, a quick search into the literature highlights that only bridgehead functionalizations, mimicking para-substitution of the benzene ring, have been reported to date (Figure $1 \mathrm{~A}$ ). This is certainly due to the absence of synthetic methods to achieve 1,2-difunctionalization of BCPs rather than the lack of desire to replace ortho- and/or metadisubstituted benzenes in bioactive molecules which has been regularly mentioned over the years. ${ }^{2}$ Recently, Mykhailiuk and co-workers reported the synthesis of bicyclo[2.1.1] the first example of saturated bioisosteres of ortho-disubstituted benzenes. ${ }^{4}$ Conversely, there is still a gap in the literature for the synthesis of 1,2-difunctionalized BCP skeletons that would act as ortho- and/or meta-disubstituted benzenes (Figure 1B). Our foray into the synthesis of such compounds started with a directed $\mathrm{C}-\mathrm{H}$ activation strategy where a bridgehead amide direction group was installed to activate the side position of the BCP. Unfortunately, all efforts towards this directed approach proved unsuccessful. While trying other tactics, we came across a paper published by the group of Schliiter in $1995 .{ }^{5}$ In their report, the authors synthesized the [1.1.1]propellane 1 bearing a methoxy methyl (MOM) protected hydroxyl group in the side chain. ${ }^{5}$ Inspired by this unexplored precedent and capitalizing on newly emerging methods for the crosscoupling of previously unreactive carboxylic acids (via decarboxylative functionalization) we report herein the development of a platform to synthesize 1,2-difunctionalized bicyclo[1.1.1]pentanes from compound 2 (Figure 1C), synthesized from 1 in 3 steps.

Figure 2 highlights the preparation of several key building blocks that allow for the preparation of various 1,2-difunctionalized BCP skeletons. Towards this end, our studies commenced by optimizing Schliiter's synthetic sequence leading to compound 1 which was obtained in $36 \%$ yield over 5 steps (Figure 2A). With compound 1 in hand, building block 2 was easily prepared in 3 steps through radical difunctionalization, ${ }^{3 d}$ deiodination, ${ }^{3 d}$ and chlorine-acetate exchange (18\% overall yield, Figure 2B). Compound 2 was chosen for the synthetic versatility it offers. Indeed, alcohol 3, carboxylic acid 4, and amine 5 were easily obtained from $\mathbf{2}$ in one, two, or three steps, respectively (Figure $2 \mathrm{C}$ ) via simple functional group manipulation. Finally, functionalization the [1.1.1]propellane 1 side chain was undertaken (Figure 2D). Radical ring opening and dehalogenation of $\mathbf{1}$ afforded compound $\mathbf{6}$ which was directly deprotected and oxidized to afford carboxylic acid 7 in $18 \%$ yield over 4 steps. The carboxylic acid moiety was the adequate handle to prepare redox active ester $\mathbf{8}$ and test its ability to undergo decarboxylative transformations. Pleasingly, compound $\mathbf{8}$ delivered borylated compound $\mathbf{9}$ in satisfactory yield under our reported 
copper-catalyzed decarboxylative borylation conditions. ${ }^{6}$ It is worth noting that compounds 8 and 9 offer limitless possibilities regarding the preparation of medicinal chemistry relevant structures. The synthesis of these 1,2-difunctionalized bicyclo[1.1.1]pentane building blocks encouraged us to examine the practicality of the platform in the context of preparing drug bioisosteres as outlined in Figure 3.

The synthesis of Sonidegib bioisostere 10 was first investigated. Starting from compound 2, MOM deprotection, followed by alcohol oxidation and ester formation afforded redox active ester $\mathbf{1 1}$ in $74 \%$ yield (Figure 3A). Subsequent decarboxylative Negishi arylation successfully delivered compound 12 in good yield. ${ }^{7}$ Finally, a simple deprotection/oxidation sequence followed by amide bond formation afforded Sonidegib bioisostere 10. A similar strategy was employed to synthesize Lomitapide bioisostere 13 from redox active ester 11 demonstrating the flexibility of the 1,2difunctionalized bicyclo[1.1.1]pentane platform (Figure 3A). Efforts then turned to the synthesis of Boscalide bioisostere 14 which bears an amide at the bridgehead position (Figure 3B). Starting from alcohol 3, a 5-step sequence afforded redox active ester 15 which smoothly underwent decarboxylative Negishi arylation to afford intermediate 16. Then, ester hydrolysis followed by Curtius rearrangement and carbamate deprotection gave the amine compound 17 in $70 \%$ yield. Final amide bond formation delivered the desired Boscalide bioisostere 14. Lastly, the versatility of the platform was demonstrated by the synthesis of Meclizine bioisosteres 18 and its reversed analog 19 (Figure 3C). From 3, a simple 4 step sequence (protection - reduction - deprotection - protection) afforded intermediate 20 bearing the methyl group at the bridgehead position. The same steps were repeated in a different order from compound 2 to afford intermediate 21 (reversed of 20) which bears the methyl group at the side chain position. Both intermediates 20 and 21 underwent amide bond coupling to obtain Meclizine bioisosteres 18 and 19 in $45 \%$ and $41 \%$ overall yield, respectively.

The scope of the 1,2-difunctionalized bicyclo[1.1.1]pentane platform was further expanded as outlined in Figure 4. Thus, compounds 22 and its reversed analog 23 were synthesized as bioisosteres of Phthalylsulfathiazole in 7 steps (34\% and $21 \%$ overall yield) from 2 (Figure 4A). In addition, compounds $\mathbf{2 4}$ and $\mathbf{2 5}$, bioisosteres of Tolvaptan, were targeted and can provide access to a total of 4 compounds to study (after separation of the diastereomers and supercritical fluid chromatography (SFC) separation of enantiomers) due to the presence of an additional chiral center (Figure 4B). Finally, a molecular matched pair synthetic campaign was undertaken to 
evaluate the usefulness of the new platform in the context of fragment-based approach (Figure 4C). Compound 26, 27, and 28 were therefore synthesized from 2, 3, and 1 in 7, 6, and 6 steps, respectively. Those simple fragments are being evaluated and should deliver precious information regarding the ortho and/or meta-character of the 1,2-difunctionalized bicyclo[1.1.1]pentane derivatives.

All of the compounds presented above are now being separated via SFC to isolate both enantiomers. Biological and computational studies are underway to validate the ortho and/or meta-character of all the aforementioned bioisosteres and results will be reported in due course.

Conclusion: For years, chemists in both industry and academia have been focusing on the incorporation of the bicyclo[1.1.1]pentane skeletons that mimic para-substituted benzenes. Herein, we reported the development of an useful platform that answers the long-standing challenge of accessing 1,2-difunctionalized bicyclo[1.1.1]pentane building blocks that could serve as ortho and/or meta-substituted benzene analogues. While the approach is admittedly lengthy, the key intermediates from which divergent synthesis can take place are easily accessed on scale and amenable to outsourcing. Future studies on more simplified access to such compounds will be pursued based on biological findings. As described above, several bioisosteres of ortho/meta disubstituted benzene containing drugs as well as simple benzene molecular matched pairs were obtained using this methodology. This initial disclosure is presented with urgency due to the demand in industry for routes to such molecules. Focus internally remains now on the biological and computational analysis of these new compounds and these results will be disclosed in due course. In the meanwhile the synthetic route delineated herein will likely be of high interest to those working in the fields of organic synthesis, agrochemistry, and/or medicinal chemistry.

Corresponding Authors: *pbaran@scripps.edu

Acknowledgment: Financial support for this work was provided by Pfizer and NIH (GM-118176). Authors are grateful to Dr. Dee-Hua Huang and Dr. Laura Pasternack (Scripps Research) for assistance with nuclear magnetic resonance (NMR) spectroscopy, to Dr. Jason Chen, Brittany Sanchez and Emily Sturgell (Scripps Automated Synthesis Facility) for assistance with HPLC, HRMS and LCMS 


\section{References:}

1. (a) F. Lovering, J. Bikker, C. Humblet, J. Med. Chem. 2009, 52, 6752-6756; (b) F. Lovering, MedChemComm 2013, 4, 515-519.

2. (a) D. C. Blakemore, L. Castro, I. Churcher, D. C. Rees, A. W. Thomas, D. M. Wilson, A. Wood, Nat. Chem. 2018, 10, 383-394.; (b) A. A. Kirichok, I. Shton, M. Kliachyna, I. Pishel, P. K. Mykhailiuk, Angew. Chem. Int. Ed. 2017, 56, 8865-8869.; (c) A. A. Kirichok, I. O. Shton, I. M. Pishel, S. A. Zozulya, P. O. Borysko, V. Kubyshkin, O. A. Zaporozhets, A. A. Tolmachev, P. K. Mykhailiuk, Chem.Eur. J. 2018, 24, 5444-5449.; (d) B. Chalyk, M. Butko, O. Yanshyna, K. Gavrilenko, T. Druzhenko, P. K. Mykhailiuk, Chem. Eur. J. 2017, 23, 16782-16786.; (e) P. K. Mykhailiuk, Org. Biomol. Chem. 2019, 17, 2839-2849.; (f) G. M. Locke, S. S. R. Bernhard, M. O. Senge, Chem. Eur. J. 2019, 25, 4590-4647.

3. For some recent examples, see: (a) R. Gianatassio, J. M. Lopchuk, J. Wang, C.-M. Pan, L. R. Malins, L. Prieto, T. A. Brandt, M. R. Collins, G. M. Gallego, N. W. Sach, J. E. Spangler, H. Zhu, J. Zhu, P. S. Baran, Science 2016, 351, 241-246.; (b) S. O. Kokhan, A. V. Tymtsunik, S. L. Grage, S. Afonin, O. Babii, M. Berditsch, A. V. Strizhak, D. Bandak, M. O. Platonov, I. V. Komarov, A. S. Ulrich, P. K. Mykhailiuk, Angew. Chem. Int. Ed. 2016, 55, 14788-14792.; (c) J. Kanazawa, K. Maeda, M. Uchiyama, J. Am. Chem. Soc. 2017, 139, 17791-17794.; (d) D. F. J. Caputo, C. Arroniz, A. B. Dürr, J. J. Mousseau, A. F. Stepan, S. J. Mansfield, E. A. Anderson, Chem. Sci. 2018, 9, 5295; (e) R. A. Shelp, P. J. Walsh, Angew. Chem. Int. Ed. 2018, 57, 15857-15861.; (f) I. S. Makarov, C. E. Brocklehurst, K. Karaghiosoff, G. Koch, P. Knochel, Angew. Chem. Int. Ed. 2017, 56, 12774-12777.; (g) R. M. Bychek, V. Hutskalova, Y. P. Bas, O. A. Zapor-ozhets, S. Zozulya, V. V. Levterov, P. K. Mykhailiuk, J. Org. Chem. 2019, 84, 15106-15117.; (h) S. Yu, C. Jing, A. Noble, V. K. Aggarwal, Angew. Chem. Int. Ed. 2020, 59, 3917-3921.; (i) Z. J. Garlets, J. N. Sanders, H. Malik, C. Gampe, K. N. Houk, H. M. L. Davies, Nat. Catal. 2020, 3, 351-357.; (j) J. H. Kim, A. Ruffoni, Y. S. S. Al-Faiyz, N. S. Sheikh, D. Leonori, Angew. Chem. Int. Ed. 2020, 59, 8225-8231.; (k) X. Zhang, R. T. Smith, C. Le, S. J. McCarver, B. T. Shireman, N. I. Carruthers, D. W. C. MacMillan, Nature 2020, 580, 220-226.

4. A. Denisenko, P. Garbuz, S. V. Shishkina, N. M. Voloshchuk, P. K. Mykhailiuk, Angew. Chem. Int. Ed. 2020, 132, 2-9.

5. R. Klopsch, A.-D. Schliiter, Tetrahedron, 1995, 51, 10491-10496.

6. J. Wang, M. Shang, H. Lundberg, K. S. Feu, S. J. Hecker, T. Qin, D. G. Blackmond, P. S. Baran, ACS Catal. 2018, 8, 9537-9542. 
7. T. Qin, J. Cornella, C. C. Li, L. R. Malins, J. T. Edwards, S. Kawamura, B. D. Maxwell, M. D. Eastgate, P. S. Baran, Science 2016, 352, 801-805.

\section{Figures:}

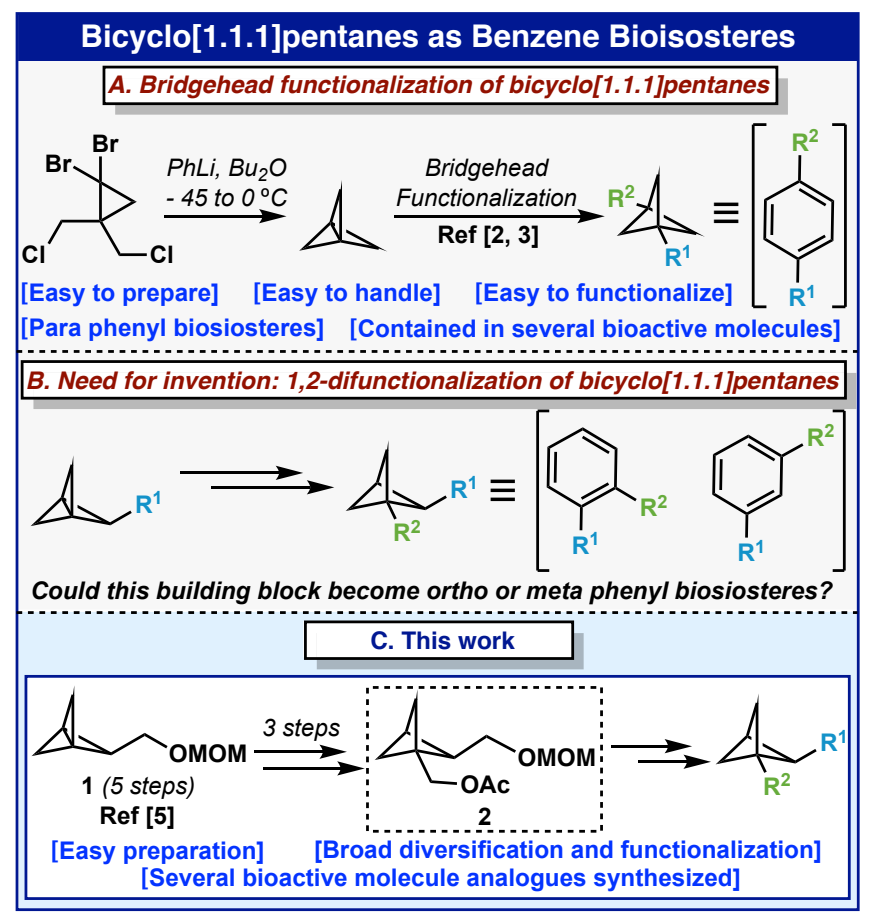

Figure 1. Introduction to bicyclo[1.1.1]pentanes as arene bioisosteres 
Preparation of Diverse 1,2-Difunctionalized Bicyclo[1.1.1]pentane Building Blocks
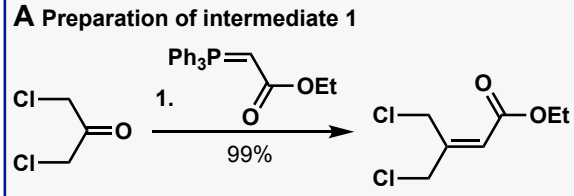

2. $\mathrm{LAH}, \mathrm{AlCl}_{3}$
$\underset{\text { 3. } \mathrm{P}_{2} \mathrm{O}_{5}, \mathrm{DMM}}{\stackrel{87 \%}{\text { (over } 2 \text { steps) }}}$
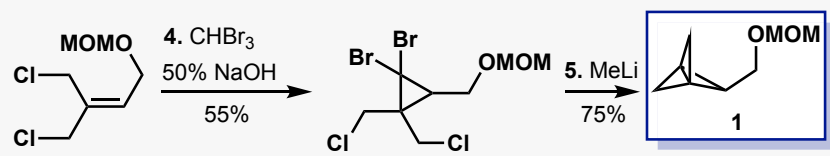

B Preparation of key building block 2

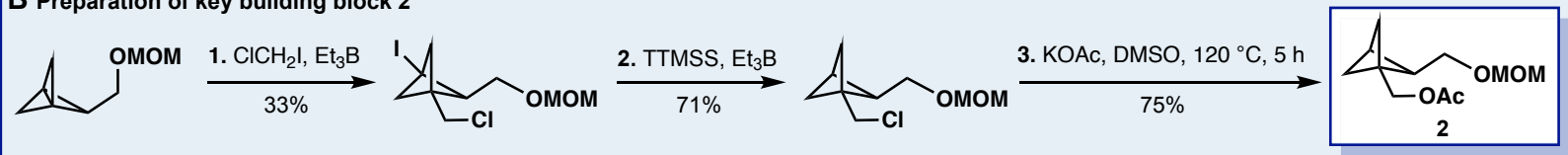

C Preparation of alcohol, carboxylic acid and amine building blocks from compound 2
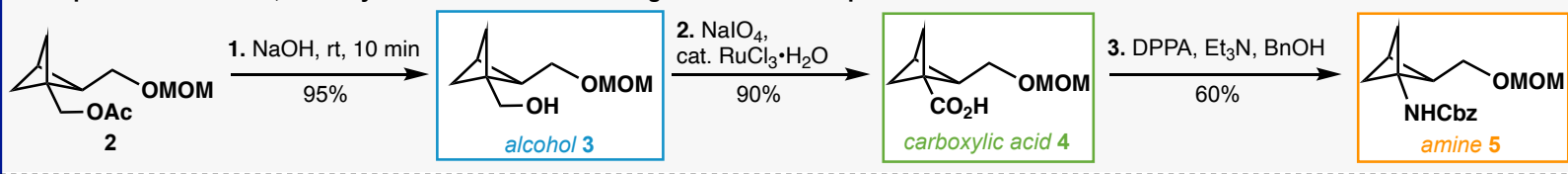

D Preparation of other useful building blocks from intermediate 1
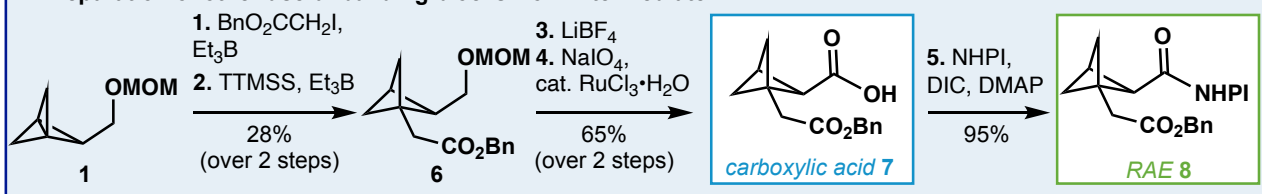

6. $\mathrm{Cu}(\mathrm{acac})_{2}$

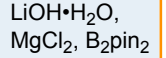
$\underset{33 \%}{\longrightarrow}$

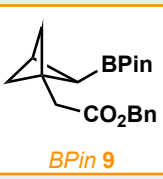

Figure 2. Preparation of diverse 1,2-difunctionalized bicyclo[1.1.1]pentane building blocks. 


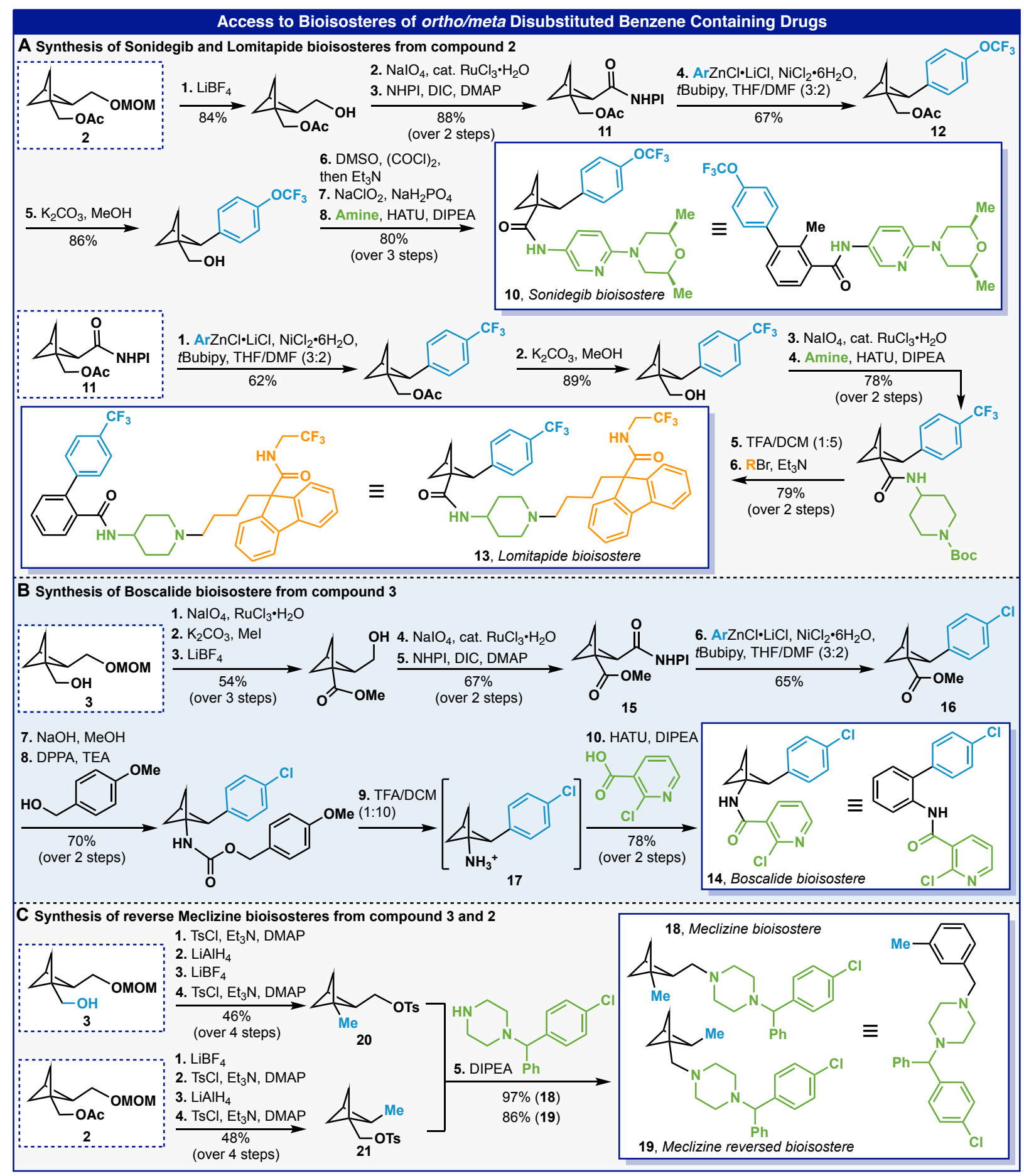

Figure 3. Access to bioisosteres of ortho/meta disubstituted arene containing drugs. 
A Phthalylsulfathiazole bioisosteres from compound 2

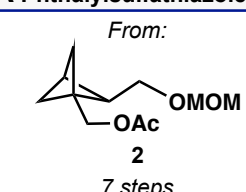

$34 \%$ and $21 \%$ overall yield

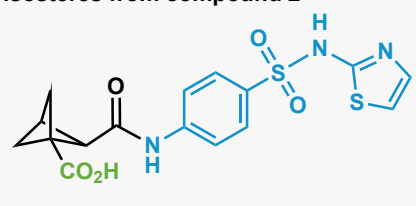

22, Phthalylsulfathiazole bioisostere

compound 2

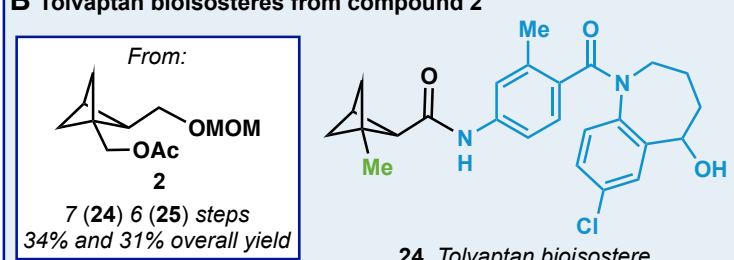

24, Tolvaptan bioisostere

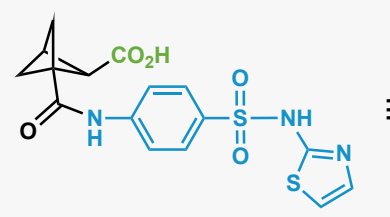

23, Phthalylsulfathiazole reversed bioisoster
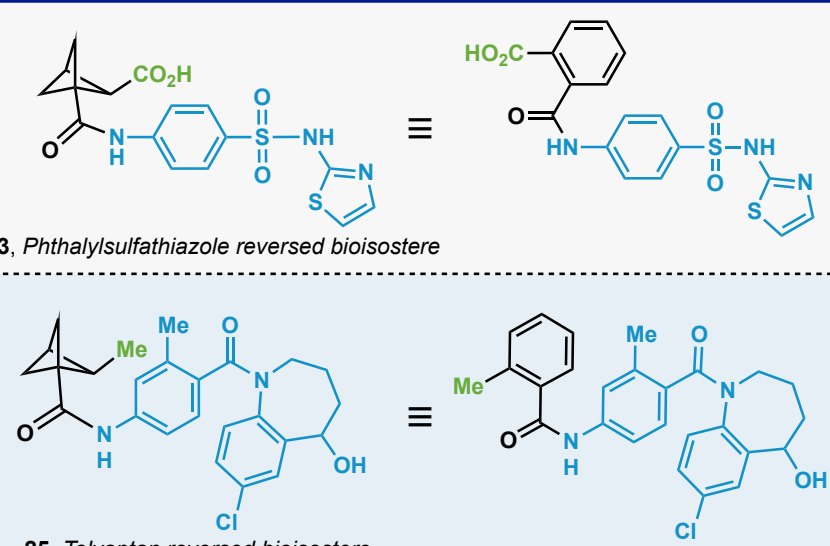

25, Tolvaptan reversed bioisostere

C Molecular Matched Pairs (MMP)

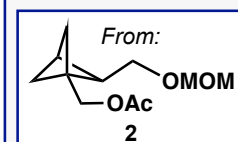

2

6 steps

$38 \%$ overall yield

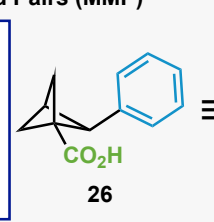

$\equiv$

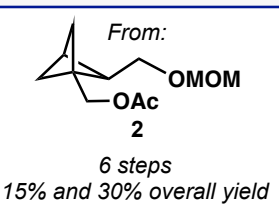

$15 \%$ and $30 \%$ overall yield

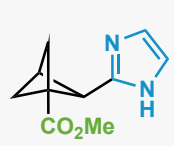

27

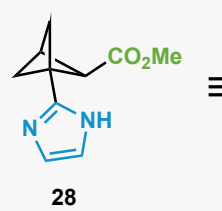

28

Figure 4. Scope expansion of the 1,2-difunctionalized bicyclo[1.1.1]pentane platform. 